\title{
Les sols (péri)urbains à l'épreuve du discours sur le primat du local
}

Discours experts, incertitudes et production d'ignorance

(Peri)urban soils in light of the primacy of the local

Élisabeth Rémy, Nolwenn Gauthier et Marine Canavese

\section{(2) OpenEdition \\ Journals}

Édition électronique

URL : http://journals.openedition.org/developpementdurable/16966

DOI : 10.4000/developpementdurable.16966

ISSN : 1772-9971

Éditeur

Association DD\&T

Référence électronique

Élisabeth Rémy, Nolwenn Gauthier et Marine Canavese, «Les sols (péri)urbains à l'épreuve du

discours sur le primat du local », Développement durable et territoires [En ligne], Vol. 11, n 1 | Avril 2020, mis en ligne le 30 avril 2020, consulté le 15 mai 2020. URL : http://journals.openedition.org/

developpementdurable/16966; DOI : https://doi.org/10.4000/developpementdurable.16966

Ce document a été généré automatiquement le 15 mai 2020.

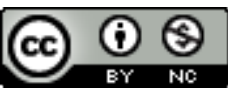

Développement Durable et Territoires est mis à disposition selon les termes de la licence Creative Commons Attribution - Pas d'Utilisation Commerciale 4.0 International. 


\title{
Les sols (péri)urbains à l'épreuve du discours sur le primat du local
}

\author{
Discours experts, incertitudes et production d'ignorance \\ (Peri)urban soils in light of the primacy of the local
}

Élisabeth Rémy, Nolwenn Gauthier et Marine Canavese

Nous tenons à remercier les relecteurs et coordinateurs de la revue pour leurs remarques et commentaires qui ont largement contribué à améliorer une première version de ce texte.

1 Le «local» constitue, depuis quelques années, un argument pour attester de la durabilité des systèmes de production alimentaires urbains. Dans un contexte de pression foncière et de renouvellement urbain se multiplient les types de supports de production pour des projets de jardinage ou « d'agriculture urbaine ${ }^{1}$ » (AU) (Duchemin, 2013). En pleine terre, sur des sols issus d'activités anthropiques et/ou des sols construits (anthroposols, technosols), sur des substrats inertes (fibres de coco par exemple) irrigués par des solutions nutritives (hydroponie) ou encore poussant dans des bassins aquacoles (aquaponie), les types de sols de l'AU sont multiples.

2 L'écho international d'expériences d'AU, comme celles en cours dans la ville de Detroit, relayées dans de nombreux médias et via des films grand public comme Demain ${ }^{2}$, a mis en avant l'effet de ces cultures sur la structuration de réseaux associatifs et la gestion par des collectifs d'habitants de délaissés urbains ${ }^{3}$. Si des recherches sur des terrains européens et états-uniens ont montré les potentialités et les limites de ces expériences en termes de justice sociale et environnementale (Paddeu, 2014; Tornaghi, 2014; Slocum et al., 2016, Darly et Demailly, 2017), on remarque que la qualité des sols n'est pas un élément abordé dans ces réflexions sur les enjeux de la territorialisation alimentaire (Mormont, 2009). Alors que des conflits d'usage ${ }^{4}$ commencent à émerger autour du développement de "l'agriculture urbaine» (Brondeau, 2017), des collectivités qui souhaitent développer ce type d'initiatives se retrouvent de plus en plus confrontées à la question de la qualité des sols.

3 La prise de conscience collective sur la contamination potentielle des sols est effectivement récente, car il n'y a encore que très peu de vulgarisation publique sur les 
sols (contrairement à l'air ou l'eau) (Ogé, 2013). Pour les spécialistes ${ }^{5}$ des sols euxmêmes, le sol urbain est encore méconnu, et pose diverses questions, notamment sur les traces des activités passées qu'il stocke, enregistre et fait parfois ressurgir lorsqu'il est manipulé (Barles et al., 1999; Morel Journel et al., 2018). Cette méconnaissance s'explique aussi par l'extrême hétérogénéité et la grande variabilité du milieu sol (Douay, 2008), sa qualité (agronomique et physico-chimique) pouvant varier du simple au triple en quelques mètres. Lorsqu'ils font des analyses, les spécialistes constatent que le sol est parfois contaminé par divers polluants (mercure, arsenic, cadmium, plomb, hydrocarbures, PCB, pesticides, etc.), et ce depuis des décennies, voire des siècles. Se pose alors avec acuité la question de la contamination (que chercher, comment ? etc.) de ces sols, laquelle fait l'objet de diverses traductions et de diffusions publiques adaptées en fonction des situations (projets de jardins collectifs, projets d'AU, recherche des polluants en amont d'un projet d'aménagement, etc.). L'exemple des sols contaminés en milieu urbain permet ainsi d'appréhender la part d'incertitudes relatives à un héritage complexe affectant le sol urbain et les limites de la publicisation des différentes incertitudes associées aux sources potentielles de contamination.

Dans une perspective quelque peu similaire, à travers cet article, nous cherchons à montrer que l'idée de maîtriser l'impact écologique des pratiques agricoles et des systèmes alimentaires urbains est liée à des discours et des pratiques qui méritent d'être interrogés, car ceux-ci conduisent à ignorer l'élément qui semblerait le plus central : la qualité du sol. En s'inspirant des apports de la sociologie des sciences et des techniques (Latour, 1989 ; Latour, 1994), on s'interroge sur les multiples traductions de l'objet sol et de ce que l'on s'autorise à faire (ou pas) en son nom; le sol (péri)urbain est-il si malléable? Que savons-nous de son histoire et de sa qualité agronomique et physico-chimique pour y projeter a priori des cultures alimentaires? Serait-il possible d'apporter quelques premiers éléments de réponse à cette question posée par $\mathrm{R}$. Proctor $^{6}$ (1991) : «Pourquoi ne savons-nous pas ce que nous ne savons pas? » Notre objectif est d'identifier les positionnements des savoirs experts (Rémy et al., 2017) impliqués dans la caractérisation du sol (péri)urbain ce qui, par extension, pose la question des vertus attribuées au local et à l'écologisation des cultures alimentaires en périphérie des villes.

5 Alors que l'on parle souvent à juste titre des processus d'invisibilisation des sols pollués, voire de pollution invisible (Zanetti et al., 2018), nous pouvons aussi nous demander quels sont les mécanismes complexes qui produisent l'ignorance (Proctor, 1995 ; Fillion E. et al., 2016) sur un nouvel objet émergent, à savoir le sol urbain nourricier ? En quoi les enjeux de relocalisation alimentaire en ville mettent-ils à jour à la fois des incertitudes liées à la qualité des sols urbains et interrogent les modes de circulation et de production de ce type de savoirs sur ces milieux ? Comment l'étude de la qualité de sols (péri)urbains peut-elle contribuer à l'analyse des mécanismes et des différentes formes que peut prendre l'ignorance, notamment sur les risques associés aux sols (péri)urbains?

6 L'écriture de cet article est alimentée par différentes enquêtes de terrain qui nous conduisent aujourd'hui à cette réflexion commune ${ }^{7}$. Ces enquêtes ont été réalisées en France sur plusieurs sites de cultures alimentaires en milieu urbain. D'une part, des recherches simultanées, menées depuis cinq ans et suivies sur un temps long, ont été faites dans les agglomérations de Lyon, Paris, Lille et Nantes ${ }^{8}$; des entretiens semidirectifs, plus récents (réalisés entre 2015 et 2018), ont également été menés auprès de 
scientifiques spécialistes des sols et d'écologie, des responsables de différents services techniques des villes et d'agents techniques de collectivités (sept entretiens), de gestionnaires impliqués sur les questions de jardinage ou d'agriculture urbaine et périurbaine dans les différentes régions étudiées. Des balades urbaines avec des habitants ainsi que des visites régulières dans ces jardins collectifs nous ont permis de compléter ces entretiens en observant les pratiques culturales des jardiniers. Des corpus d'archives, personnelles et publiques, ont également été étudiés (Canavese et Frioux, 2019). Plus récemment, une attention particulière a été portée au traitement médiatique de l'AU et des systèmes alimentaires urbains, tant dans la presse nationale que locale, généraliste et spécialisée. Sans oublier le suivi et les observations régulières de réunions publiques, de processus de concertation concernant l'élaboration de plans alimentaires territoriaux (à Paris et sa région), de conseils de quartiers et de situations d'expertises. En somme, il s'agit ici d'une forme de retour d'expérience, d'un recul pris sur nos terrains pour tirer les premiers enseignements de diverses études et observations que nous avons rassemblées sur le sujet depuis 2014.

7 Dans une première partie, nous interrogeons les effets du développement de l'AU sur la construction de plusieurs figures de sols urbains nourriciers - dans le sol en place, en hors-sol ou sans sol. Ces différentes configurations contribuent-elles à une meilleure connaissance des sols auprès du grand public ou au contraire à produire de la confusion? Dans un second temps, nous proposons une discussion sur la relation entre qualité et localisation des productions distribuées en circuits courts et de proximité ${ }^{9} \mathrm{Si}$ le lieu de production prend le pas sur le support de production dans la représentation de la qualité des produits, en quoi la question de la qualité des sols peut-elle participer à nuancer les discours performatifs concernant les effets de la relocalisation comme forme d'écologisation des pratiques?

8 Enfin, en croisant les apports de la littérature sur la production de l'ignorance avec ceux d'Ulrich Beck (2001), une discussion plus large est lancée sur la dialectique de production de savoirs experts et d'ignorance à propos de la nature des sols (péri)urbains et de leurs risques potentiels. À travers ces débats et controverses ${ }^{10}$ émerge la question de l'intentionnalité (Jouzel et Dedieu, 2013; Chabbal, 2005) des acteurs concernant ce que l'on sait (ou pas) sur ces sols et ce qui est partagé ou ne l'est pas. Pourrait-on s'attendre à de possibles « effets boomerangs ${ }^{11}$ » (Beck, 2001) lors du développement des cultures alimentaires sur ces sols urbains et péri-urbains valorisés au nom du primat du local?

\section{Sols méconnus, une fabrique urbaine source d'ignorance}

9 L'entrée en politique du sol urbain se réalise selon différentes représentations des technosciences ${ }^{12}$ et de leur capacité supposée à participer à l'écologisation du système alimentaire urbain $^{13}$. Le cas parisien est intéressant à citer car, sous l'impulsion associant étroitement une politique volontariste et une pratique ingénieuriale, il concentre environ $50 \%$ des projets d'AU au niveau national ${ }^{14}$.Cette politique relative au développement de «l'agriculture urbaine» repose sur deux «fictions institutionnalisées" (Jouzel et Dedieu, 2013): le local est un gage de qualité des produits cultivés; le sol urbain est apte à recevoir des cultures à vocation alimentaire. À travers divers exemples, il s'agit d'expliciter comment s'organise ce mouvement 
autour du savoir expert et, parfois, de la production de l'ignorance vis-à-vis des sols urbains. L'écologisation en tant qu' entreprise de recadrage cognitif et normatif " (Ginelli, 2017), par des acteurs institutionnels et/ou mandatés concernés par des enjeux communs (Ginelli, 2012), s'appuie sur des discours (Gautier et Benjaminsen, 2012) qui opèrent une recomposition entre connaissances des milieux de production et pouvoir de normalisation des pratiques culturales. Dans cet article, l'attention est portée sur le rôle de l'expertise dans un contexte marqué par la mise en place de projets d'AU ou de jardins collectifs assignant un nouveau rôle au sol urbain, celui de sol nourricier. Sera analysée ici l'idée d'un double mouvement concomitant et contradictoire : d'un côté, la difficulté à parler du sol et surtout de sa contamination potentielle (sauf cas extrêmes et médiatisés ${ }^{15}$ ) ; et de l'autre, le rôle joué par les récits du « nourrir la ville sur la ville » via la diffusion médiatique d'expériences d'AU et l'effet performatif que produit, en retour, ce discours médiatique enchanté sur les vertus attribuées aux sols (péri)urbains.

\subsection{Sol (péri)urbain et pollutions diffuses : une définition feutrée pour un objet de connaissance confiné}

10 La façon de composer avec l'incertitude influe lourdement sur la caractérisation du sol urbain et sur les rôles qu'on lui attribue. Pendant longtemps et encore actuellement, il reste difficile de s'exprimer publiquement sur la contamination du sol ${ }^{16}$.Ce constat résulte d'une analyse des discours et des pratiques relatives aux projets de création de potagers urbains, d'aménagement d'espaces verts ou de «renaturation» de friches industrielles.

11 Cela commence par l'usage de formules rhétoriques. Lors d'une réunion publique en 2017 concernant un jardin collectif pour lequel des analyses de sols ont montré une contamination, un expert en science du sol explique que le « sol est enrichi en plomb et en cadmium » pour signifier que la teneur élevée de ces éléments en fait un sol contaminé. Des jardiniers se sont ensuite exprimés sur l'incompréhension engendrée par l'utilisation de ce terme, comme cette personne jardinant depuis sept ans : «Je ne savais pas que le sol pouvait contenir des métaux. Quand le scientifique a dit que le sol était enrichi,j'ai pensé que c'était une bonne nouvelle. »Ce type de phrase peut en effet prêter à confusion dans la mesure où la notion "d'enrichissement" a une connotation positive. Cette notion d'enrichissement se décline autrement dans une exposition intitulée "Capital Agricole » qui s'est déroulée récemment à Paris ${ }^{17}$. Sur un panneau de l'exposition, on peut lire que "Paris est un ventre vorace aux besoins toujours croissants. Demandeur d'agricole, l'urbain assure les lieux de transformation - abattoirs, tanneries, moulins [...]. Les cultivateurs remportent des immondices de toutes sortes pour enrichir les terres de banlieue ». Porté principalement par des architectes, paysagistes et urbanistes, ce récit fondateur de «l'urbanisme agricole » décrit ainsi une vision idyllique de la ville, du recyclage et de ses relations à ses terres périphériques, bien éloignée de la description de la « ville délétère » (Barles, 1999). Dans son ouvrage éponyme, Sabine Barles parle en effet de " champs d'épandage qui apparaissent comme des marais artificiels dignes des palus du XVIII ${ }^{e}$ siècle et suscitent une vive polémique » (p. 11) et ceci à partir des années 1850 - nous y reviendrons plus loin dans ce texte.

Nous avons enquêté auprès de services techniques de mairies dans lesquelles différentes stratégies politiques ont pu être choisies : la communication des pollutions aux jardiniers, ou au contraire le refus de s'exprimer publiquement, ou alors 
partiellement sur la question (Canavese, 2015). En ce sens, des informations concernant des sites pollués se disent de manière si feutrée que seules les personnes les plus averties peuvent prendre la mesure du problème. Il en résulte une non-sensibilisation des habitants sur la question des contaminations du sol où l'ignorance sur le sujet, sans être totalement cachée, n'est jamais explicitée en détail. C'est ce qu'exprime un responsable en aménagement du territoire confronté à la gestion d'un sol pollué lors d'un entretien réalisé en 2017 :

13 «Par rapport à la population effectivement, on ne leur a jamais dit qu'on a neutralisé les risques. Là, je suis d'accord avec vous, mais on ne peut pas dire qu'on ment aux populations, on s'arrange pour qu'ils n'aient pas bien compris l'information jusqu'au bout ou on essaie de répondre à un problème techniquement et financièrement acceptable. »

14 Ces manières de s'adresser au public dessinent en creux la figure du public que soustendent ces discours: ces derniers lui attribuent l'incapacité à gérer ce type d'informations et l'incertitude propre au sol (péri)urbain en considérant ces informations comme " anxiogènes ${ }^{18}$ » - expression maintes fois entendue durant nos entretiens. Peu informé, le public se voit alors qualifié, non sans paradoxe, de «nonsachant ». Cette opacité est entretenue par le fait que la sensibilité profane, si elle est capable d'évaluer (en partie) la fertilité d'un sol ou la présence de certains polluants visibles (traces d'hydrocarbures), demeure impuissante à saisir les pollutions invisibles et inodores (arsenic, cadmium, plomb, mercure, etc.) qui nécessitent, quant à elles, la médiation d'un instrument (Grenet et al., 2016).

Des débats autour de la qualité des sols existent au sein de communautés scientifiques et savantes, telle que l'Association française de l'étude des sols qui rassemble des scientifiques et des praticiens spécialisés sur l'étude des sols (chercheurs, enseignants, étudiants, techniciens, agriculteurs...). Pour autant, ces controverses donnent rarement lieu à des communications vers le grand public. En revanche, les informations circulant dans l'espace public concernant l'absence de risque de l'agriculture réalisée sur des sols urbains ou hors sol, doivent être relues au prisme de la multi-positionnalité de ses porte-parole.

16 Si la question des pollutions des sols urbains et périurbains commence à être débattue par divers spécialistes (essentiellement géochimiste, sciences du sol, pédologues, écotoxicologues), la communication en direction du grand public reste délicate. Car, dans le même temps, d'autres acteurs scientifiques jouent un rôle d'experts de l'aménagement urbain (très proches des cabinets politiques des collectivités) et adoptent, en jouant sur diverses casquettes (Ronsin, 2018), un positionnement fort différent; à l'appui de bureaux d'études créés ad hoc, leur démarche vise en effet à accompagner le développement de start-up en $\mathrm{AU}^{19}$, ce qui a pour conséquence de minorer les risques associés au milieu urbain. Pour illustrer notre propos, donnons un exemple: ces derniers cherchent à imposer leurs propres conseils comme le port de gants ou de masque pour inciter à développer une filière agricole sur des sols (péri)urbains reconnus comme plus impactés que les sols agricoles habituels, et sans prévenir au préalable le futur consommateur. Nous reviendrons à la fin du texte sur la pertinence de ce type de préconisations qui ne tiennent pas compte des impasses auxquelles elles ont déjà donné lieu et les effets produits en termes de production de l'ignorance sur les potentialités de ces sols.

17 La porosité entre expertise, science et action dans ce domaine produit alors de la confusion quant aux caractères scientifiques des arguments mobilisés pour attester de 
l'absence de risque. Comme le souligne Girel à propos des stratégies agnotologiques «ce n'est pas parce que l'on parle de science que l'on fait de la science» (Girel, 2013). Car, c'est bien vers des questions éthiques et déontologiques (Coutellec, 2015) que pointe notre analyse par rapport aux conditions d'émergence de la figure des sols urbains nourriciers. De plus, la réduction de la question de la maîtrise de ces risques à un arbitrage qui serait " purement» technique tend à évacuer la portée politique de ces débats, qui concernent pourtant la problématique collective de l'organisation des villes, des systèmes alimentaires et des territoires, point que nous discuterons plus largement dans la seconde partie de ce texte.

Le sol est ainsi écartelé entre la parole feutrée et contrôlée des autorités technicoadministratives et le fracas - relatif - des rares associations militantes sur les sites et sols pollués. L'association les Robins des bois publie par exemple L'Atlas de la France toxique $(2016)^{20}$, un ouvrage qui regroupe plusieurs cartographies d'espaces pollués sur l'ensemble du territoire. Par-delà ces deux positions extrêmes, comment le sol urbain nourricier est-il mis en scène dans certains médias?

\subsection{La mise en scène du sol urbain nourricier : ce que l'on proclame et ce que l'on passe sous silence}

19 Ces dernières années, de nombreux articles dans la presse nationale et locale ont relaté le fleurissement d'expériences d'AU. Relayant les argumentaires des entrepreneurs et des collectivités engagées dans le développement de l'AU, nombre de ces articles ont contribué à la mise en scène d'un sol urbain nourricier ${ }^{21}$. Dans leur ouvrage Les marchands de doute, N. Oreskes et E. Conway (2012) retracent le rôle des médias dans la diffusion de certains arguments revêtant les habits de la science, mais qui servent avant tout des objectifs politiques et économiques ${ }^{22}$. Pour autant, d'autres articles se font écho de débats concernant les promesses de l'AU. Certains nuancent fortement les possibilités d'autosuffisance alimentaire offertes par ces modèles de production et interrogent le potentiel alternatif d'initiatives coûteuses et au modèle économique incertain, enfin, d'autres publications soulignent les ambiguïtés des modèles de production hors sol au regard de leur coût énergétique ${ }^{23}$.

20 Si une partie de la presse a discuté du développement de l'agriculture urbaine, c'est également sur les réseaux sociaux que se déroule une part importante de la diffusion de ces expériences. Largement investis par des entrepreneurs et des start-up associant innovations numériques et productions alimentaires, ces réseaux sont des supports pour la communication d'histoires qui enchantent l'urbain par l'alimentaire: des fraises "produites à Paris» dans des containers, des champignons dans un parking souterrain, des micro-pousses cultivées en bacs sur des espaces en friches. Les équipes de communication de ces entreprises élaborent des récits valorisant la proximité du consommateur au lieu de production et au producteur comme élément de la valeur du produit. La communication, à travers notamment des vidéos accessibles librement, donne à connaître les procédés de culture en même temps qu'elle explicite les valeurs environnementales que cherchent à incarner ces entreprises, en s'abstrayant de la culture du sol notamment ${ }^{24}$. L'absence d'emploi d'intrants chimiques et d'OGM, souvent affichés sur les emballages, est un autre élément qui doit conférer sa qualité au produit, évacuant le risque de pratiques attestées comme nocives par ailleurs. Si les types de supports de culture sont présentés (marc de café, composts, solutions nutritives 
contrôlées, etc.), ils apparaissent dans l'argumentation en tant que bénéfices: en particulier des solutions de recyclage de déchets urbains, inscrivant ces expériences dans une dynamique d'économie circulaire ${ }^{25}$. En attestant d'une certaine transparence sur les procédés de production et les matériaux employés, ces entreprises assurent contrôler la qualité au moyen de procédés techniques non nocifs pour l'environnement urbain et les aliments. Cependant, les incertitudes concernant l'emploi et la provenance de ces supports de production sur la qualité des produits ne font pas l'objet d'explicitation.

21 La communication focalisée sur des aspects particuliers des connaissances, présentées comme confirmées techniquement, n'aborde pas d'autres aspects relatifs à la complexité de produire de l'alimentaire en ville. Comme le souligne U. Beck: «À cet égard, la société du risque est aussi la société de la science, celle des médias et de l'information. En son sein se forgent de nouvelles oppositions entre ceux qui produisent les définitions $d u$ risque et ceux qui les consomment" $(2001: 84)$. En ce sens, la perspective enthousiasmante de "nourrir la ville sur la ville" oriente le regard vers ce que donnent à voir ces expériences d'AU, pour autant elle ne rend pas visibles les incertitudes sur le milieu urbain. Comme le souligne M. Girel à propos des apports d'U. Beck sur la non-connaissance comme caractéristique de la modernité: "Les effets inattendus de la modernisation peuvent être conçus comme une manifestation d'une nonconnaissance croissante » (Girel, $2017: 28$ ).

Dans un contexte marqué par l'intervention de promoteurs de l'agriculture urbaine, tant des entrepreneurs que des collectivités ou des scientifiques dans l'aménagement urbain, comment le milieu sol est-il appréhendé et présenté aux citadins et aux jardiniers?

\subsection{Les paradoxes associés aux cultures sur sols (péri)urbains}

«L'agriculture urbaine » revêt plusieurs formes et se définit selon différents modèles et pratiques culturales, que l'on choisit ici de différencier en fonction de leur rapport au sol. Puisque l'AU cherche à intégrer des sols fort différents, ces derniers ne posent pas les mêmes questions en termes de circulations des savoirs experts sur la nature et la qualité de ces sols.

On peut ainsi distinguer les cultures en pleine terre (jardinage, maraîchage), le horssol, en bac par exemple, où l'on utilise des substrats de culture (tourbe, compost ${ }^{26}$, terreau, etc.), et les cultures qui se passent totalement de sols, comme l'hydroponie (culture sans support de culture, et fertilisation de la plante par des solutions nutritives) ou l'aquaponie (culture en circuit fermé dans lequel la plante est alimentée en nutriments par le biais de bassins aquacoles dans lesquels sont élevés des poissons également destinés à la consommation). Pourtant, de récentes études sur la qualité des productions urbaines réalisées à la demande d'entreprises professionnelles d'AU (Chapelle, 2013), ou d'organismes de défense de l'environnement attestent d'une certaine connaissance par les acteurs de l'AU de la pollution des sols. Pour autant, les discours à l'attention des jardiniers et/ou des consommateurs sur la qualité des produits n'intègrent pas cette dimension dans toute sa complexité.

Par exemple, des entreprises d'agriculture urbaine lauréates de dispositifs visant à encourager "l'agriculture urbaine", à l'instar des Parisculteurs ${ }^{27}$ certifient ainsi l'absence d'OGM et l'emploi de pesticides dans leur culture, opérant de cette manière 
une distinction vis-à-vis de pratiques agricoles nocives et largement décriées aujourd'hui. Pourtant, le milieu urbain (eau, air et ici le sol) pose des questions sur le plan écologique. Nous pouvons présenter plusieurs paradoxes allant dans ce sens.

En ce qui concerne la culture des sols en place, comme dans le cas de jardins collectifs (les plus anciens), de nombreux sols sont encore aujourd'hui méconnus. Il faudrait en effet un nombre considérable d'études pour vérifier la qualité des sols et des végétaux produits dans ces nombreux jardins partagés ou familiaux (ex-jardins ouvriers) qui ont été implantés sur des délaissés urbains ou le long des infrastructures routières ou ferroviaires. Le retour sur une décision reste difficile à réaliser (Chateauraynaud et al., 1999) en pratique, puisque la décision d'aujourd'hui peut discréditer la gestion d'hier, empêchant ainsi la révision des avis ou de ce qu'on a laissé faire. Actuellement, une distance d'évitement est difficile à appliquer rétroactivement (notamment pour des questions de responsabilités) alors que des précautions peuvent être prises lors de la création de nouveaux jardins. Une fois encore, la lecture d'U. Beck éclaire notre propos. Rédigeant son ouvrage La société du risque dès 1986 , le sociologue fait le constat suivant qui montre l'ampleur du décalage contemporain en termes de projections sur les sols anthropisés: "Les zones urbaines et industrielles, les autoroutes et les grandes artères de circulation polluent la terre autour d'elles. Même si l'on débat encore de savoir si la proportion du territoire de la République fédérale d'Allemagne qui est polluée de la sorte est déjà de $7 \%$ ou si nous atteindrons ce chiffre très prochainement, il est clair que l'on ne peut plus envisager sérieusement l'exploitation agricole de ces $z^{2} e^{28}{ }^{28}$ " Le principe est le même: le patrimoine est dévalué, on observe un phénomène rampant $d$ '" expropriation écologique "(p.69). Que dire alors d'un projet de «ferme autoroutière» envisagée le long de l'autoroute A1 et présentée dans le cadre l'exposition «Capital agricole»? L'idéalisation du sol (péri)urbain est ici particulièrement prégnante car, et ce n'est pas le moindre des paradoxes, ces promoteurs (architectes, urbanistes et certains experts agronomes) envisagent de se réapproprier ces zones polluées pour des cultures alimentaires, allant à l'encontre des experts précautionneux travaillant sur les risques liés à ces sols (sciences du sol, géochimistes). Ces derniers prônent l'instauration d'une distance d'évitement d'au moins 30 mètres des infrastructures de transport (Branchu, 2013) ${ }^{29}$. On assiste ici à une lutte épistémique sur la définition des potentialités attribuées au sol. Comment le citadin lambda peut-il s'y retrouver dans ces injonctions contradictoires? Ces réappropriations de délaissés urbains autoroutiers sont-elles écologiques?

Par ailleurs, les "porteurs de projets » de jardins collectifs défendent l'idée qu'ils cultivent des légumes et des fruits sans pesticides sachant que les consommateurs y sont sensibles, alors que pendant des décennies ces pesticides ont été - et sont encore parfois - épandus sur les sols de jardins. Des pesticides sont donc présents pour de longues années encore dans ces sols, avec le souci que les produits de dégradation de ces molécules sont parfois plus dangereux que les molécules d'origine (Tixier et al., 2002). De plus, l'impact des pesticides dans les sols et les végétaux des jardins collectifs est encore peu étudié (Jacquier, 2018). Tout ceci participe à la production de l'ignorance sur la capacité du milieu (péri)urbain à accueillir des cultures alimentaires. Ce sont les pratiques qui sont rendues visibles, la qualité des sols est parfois occultée sans prendre en compte le ou les héritages (déchets, impacts agricoles, industriels, etc.) qui, sur un temps long, lui donnent sa qualité ou sa nocivité potentielle. La situation est donc bien 
plus complexe qu'elle n'y paraît et nécessite d'étudier les sites, au cas par cas, sans dogmatisme ni aveuglement.

Plus indirectement, c'est également au niveau de l'aménagement de ces jardins collectifs que la confusion peut se créer quant à la compréhension de ce qu'est un sol urbain. Alors que les citadins renouent, depuis les années 2000 avec le jardinage en ville (Dubost, 1997 ; Demailly, 2014 ; Brondeau, 2017, op. cit.), ce développement du jardinage se réalise-t-il concrètement dans le "sol urbain"? Prenons l'exemple des aménagements que des services techniques ont été obligés de créer suite à la forte demande du cabinet politique de la Ville en charge de la biodiversité, de développer des jardins collectifs au sein de leurs espaces verts. À première vue, il s'agit de créer un jardin dans un parc urbain. Sauf que ce dernier a été conçu au-dessus de l'emplacement d'une ancienne usine de $\operatorname{gaz}^{30} .$. Ce projet a été mené contre l'avis des services techniques municipaux, car leur étude historique concluait à une suspicion lourde de pollution volatile ${ }^{31}$, liée au passé industriel du site. Dans de telles circonstances, le dispositif d'aménagement consiste à excaver la terre d'origine sur 30 à 60 centimètres, puis à rapporter de la "terre végétale " par-dessus une couche drainante destinée à empêcher les racines d'aller prospecter au-delà de l'horizon "sain ». Ce jardin a même fait l'objet d'un dispositif encore plus protecteur qui s'est traduit, compte tenu des circonstances, par la mise en place d'une géomembrane imperméable qui isole hermétiquement la terre d'apport ${ }^{32} \mathrm{du}$ sol en place. La réalisation du jardin est le fruit d'une injonction politique conduisant ainsi à invisibiliser l'histoire industrielle du site et son héritage environnemental. Différentes questions se posent alors sur la façon d'appréhender et d'aménager le sol en ville. Décrivant le dispositif renforcé installé ici, un responsable du service d'aménagement des espaces verts explique «[...] ce n'est plus un système drainant qui a été mis en place, c'est un système d'étanchéité globale, c'est une géomembrane $e^{33}$ imperméable à tout. C'est une mare comblée, c'est vraiment l'extrême, on n'avait jamais vu ça avant [...] C'est complètement artificiel, complètement déconnecté $d u$ sol $^{34} »$. La réponse à cette pollution par la mise en place d'un dispositif technique a un impact cognitif. Concernant la perception du sol, on crée par ce dispositif une sorte de «trompe-l'œil » pouvant donner l'impression, si on n'a pas suivi les étapes de création du jardin ${ }^{35}$, de cultiver véritablement dans le sol urbain, alors que cet aménagement s'apparente davantage à une sorte de " hors-sol enterré3 ${ }^{36}$ ».

Ailleurs, ce sont des bacs que les promoteurs de l'agriculture dite urbaine posent sur une ancienne friche industrielle non dépolluée. On cultive dans des bacs hors sol, ce qui suscite bon nombre de questions : quelle est la constitution de ces substrats et sont-ils durables? Est-on certain qu'il n'y a pas d'ingestions ni de réenvol de poussières contaminées (Douay, op. cit.) sur les sites pollués accueillant ces bacs? Qu'en est-il des terres sorties du site lorsqu'elles sont, par exemple, collées aux chaussures des jardiniers? Ces pratiques ne sont pas anodines, car elles conduisent à des pertes de repères sur le sol en ville et en proche banlieue laissant penser que tous les espaces urbains, pourtant maintes fois remaniés, seraient cultivables pour l'alimentation.

En conséquence, le lien au sol constitue à notre sens un élément charnière pour l'analyse de l'écologisation des systèmes alimentaires. Il est pourtant peu analysé dans ses dimensions historiques, politiques et socio-techniques. Dans cette perspective, il s'agit de mettre en évidence des mécanismes qui permettent d'expliciter l'invisibilité $\mathrm{du}$ sol et de ses contaminations dans les questionnements autour de la mise en culture des espaces urbains. Alors que persiste la difficulté à s'exprimer publiquement sur les 
contaminations que peuvent receler les sols et les espaces (péri)urbains ${ }^{37}$, leur utilisation à des fins alimentaires devient un vecteur de marketing territorial ${ }^{38}$.

\section{Le local et la ville durable à l'épreuve du sol urbain nourricier}

31 Le sol urbain nourricier est, comme nous l'avons indiqué dans la première partie, dans de nombreux cas d'AU « hors sol ». Partant, la mise en avant de l'AU comme alternative aux techniques agro-industrielles ne se révèle-t-elle pas un leurre? Quelle place tiennent ces expériences dans la production discursive sur l'écologisation des pratiques de l'aménagement urbain? Telles sont les principales questions qui organisent cette seconde partie de discussion.

\subsection{S'émanciper du sol : une voie d'avenir pour l'agriculture durable?}

À travers les débats que nous avons présentés à propos des incertitudes concernant la qualification du sol urbain se pose une question d'aménagement de la ville et de sa périphérie. La diffusion de l'AU pourrait-elle contribuer à la consommation des espaces agricoles périurbains? Le développement d'une forme d'ingénierie agronomique pour la construction de sols fertiles questionne les futurs usages de ces dispositifs techniques. Est-ce aux seuls experts des technosciences et aménageurs de décider de ces usages? Cette réflexion d'un pédologue illustre bien les termes du débat : « On peut aussi essayer de rendre service aux sols en n'appréciant pas que l'Agro et l'Inra détruisent des hectares des meilleurs sols de France sur le plateau de Saclay pour installer la future AgroParisTech et faire pousser sur les toits de ladite Agro des citrouilles géantes en "compensation" écosystémique ${ }^{39} . »$

Les formes d'agriculture qui se développent en ville s'insèrent dans une évolution plus large de l'agriculture. Certains types de fermes urbaines, à l'instar des fermes verticales et des projets de tours maraîchères, seraient l'« image paroxystique » des mutations en cours dans les mondes agricoles et dans les rapports de l'agriculture à la ville (Purseigle et al., 2012). Nous ne présenterons pas ici les modèles économiques sur lesquels elles reposent. Notre regard porte surtout sur ces nouveaux modèles de culture hors sol qui, en mobilisant des technologies de précision, sont censés participer à l'écologisation des pratiques agricoles. Pourtant ces derniers n'en sont pas moins intégrés à un système mondialisé de circulation des ressources naturelles. À titre d'exemple, les intrants employés dans les solutions hydroponiques des fermes verticales sont constitués notamment de phosphate et de potasse, dont la provenance est rarement indiquée, mais dont on suppose l'extraction minière (parfois lointaine), remettant ainsi fortement en cause le primat du local. De fait, un document récent de l'Ademe (2017), portant sur la durabilité de l'agriculture urbaine, pointe les limites de ces modes de production en termes de consommation d'eau et d'énergie, notamment pour les projets développant les cultures sous led, allant jusqu'à argumenter que "l'augmentation des impacts dans d'autres aspects peut annuler les avantages de la réduction des transports » (p. 9). Bien qu'il s'agisse d'arguments largement mis en avant par les promoteurs de l'AU, ce rapport souligne au contraire la contribution réduite des formes les plus technologiques de ces fermes à la durabilité des systèmes urbains. Fonctionnant en 
circuits clos et contrôlés, elles ne contribuent donc que de loin à la végétalisation des villes. Dans certains cas, leur activité va au-delà de l'agriculture urbaine et consiste davantage à l'élaboration de modèles logistiques, comme c'est le cas de la Lufa Farms à Montréa ${ }^{40}$. La Lufa Farms est une entreprise qui se présente comme étant la première au monde à avoir implanté une ferme sur un toit (en 2011). L'entreprise propose la vente d'une gamme de légumes feuilles, de quelques légumes fruits comme des poivrons et des tomates, des légumes racines (betteraves) et à bulbes (oignons) ainsi que des plantes aromatiques, produits en hydroponie et sur substrats sur trois toits à Montréal et dans sa périphérie. Cependant, le reste des produits proposés est issu de partenariats conclus avec des producteurs (en pleine terre) situés dans la grande couronne de Montréal.

Pour s'adapter aux contraintes d'espace et de disponibilité du foncier en milieu urbain dense, tout en s'émancipant des contraintes de la culture en pleine terre (qualité des sols, parasites, etc.), les promoteurs de modèles de "fermes urbaines" hors sol, ou autrement nommés "indoor farming ${ }^{41}$ » développent des techniques de production hors sol telles que l'aquaponie, l'hydroponie, ou encore font usage de lumières artificielles pour remplacer les rayons du soleil. Un entrepreneur qui a développé un système de production hors sol entièrement informatisé et sous lumière artificielle destiné à être installé au cœur des villes est interviewé dans l'émission Vox Pop. Il s'exprime en ces termes: "Nous développons un système pour faire pousser les plantes sans pesticide ni herbicide. C'est une nourriture extra-saine, elle n'a pas besoin d'être lavée, elle peut être consommée immédiatement [...] avec la machine on devient des agriculteurs 2.0, cela peut même être vous derrière votre ordinateur, dans votre bureau ${ }^{42}$." À l'image de cette expérience, ces entreprises semblent vouloir signifier que la transition vers des systèmes alimentaires écologiques correspond à l'investissement des espaces géographiques au plus proche des consommateurs. La représentation d'une production au plus près des consommateurs semble supplanter une approche holistique des impacts écologiques de l'ensemble du système de production.

Pour reprendre l'exemple de l'entreprise montréalaise citée plus haut, ses fondateurs assurent qu'en suivant leur modèle, "la conversion de 19 toits de centres commerciaux de taille moyenne permettrait l'autosuffisance de Montréal en matière de verdures ${ }^{43}$ ». Ces ordres de grandeur correspondraient à un calcul de production par comparaison avec une production en pleine terre, pour le même nombre de plants. De nombreuses entreprises et start-up spécialisées dans l'AU véhiculent ce discours selon lequel l'agriculture sur les toits participerait à la protection des sols non urbanisés. Il est utile de rappeler qu'à l'heure actuelle aucune étude économique fiable n'a encore été menée pour valider ou non les rendements annoncés par les entrepreneurs, et que pour le moment les projets d'AU sont majoritairement financés par des fondations, partenaires privés, acteurs publics, etc., soucieux de soutenir des innovations alimentaires considérées comme durables (Ademe, 2017).

De cette manière, ce type de discours techniques participe à construire des critères de performance associés aux enjeux d'écologisation des pratiques de productions agricoles qui ont un impact cognitif sur ce que l'on considère comme une " agriculture durable ». Par exemple, l'entreprise Peas\&love qui propose la location de potagers sur des toits en intra-urbain, dans les régions parisienne et de Bruxelles, met en avant des pratiques culturales « en mode biologique ${ }^{44}$ » et a choisi « un mode d'agriculture biologique et verticale hors sol sur substrat ». Or, la mise sur le même plan de ce type de production hors sol 
avec les principes de l'agriculture biologique fait l'objet de débats ${ }^{45}$. C'était le sens de la proposition de pays scandinaves et baltes en 2017 d'inscrire dans le cahier des charges européen de l'agriculture biologique la possibilité de cultiver en bacs. S'émanciper des sols reviendrait à mettre en cause l'un des piliers de la bio, à savoir la préservation des sols. La réglementation de l'agriculture biologique de 2009 interdit formellement dans son article 4 la « production hydroponique ${ }^{46} »$. Cette proposition a soulevé les oppositions de plusieurs organisations biologiques en France, conjointement avec les chambres d'agriculture, qui ont rappelé dans un communiqué de presse les principes de l'agriculture biologique : la rotation des cultures et le lien au sol ${ }^{47}$.

\subsection{Un « retour au local » comme garantie de qualité alimentaire ?}

D'un point de vue géographique, la proximité est-elle forcément un gage de qualité des produits cultivés compte tenu des contaminations potentielles des sols urbains? Chemin faisant, la conception que l'on se fait du sol urbain et de l'héritage qu'on lui attribue (ou non) conduit là aussi à questionner ce «local». Comme le soulignent Adrien Baysse-Layné et Coline Perrin, «les circuits "relocalisés" du début du XXle siècle se construisent en partie autour de l'idée du retour à un "local" considéré comme échelle naturelle de l'approvisionnement alimentaire : en "relocalisant", les initiatives actuelles renoueraient avec des schèmes spatiaux antérieurs » (2017). Or, ces deux auteurs constatent que dans le cas d'une ville moyenne comme Millau, leur cas d'étude, la valorisation de nouvelles formes d'approvisionnement de proximité ne correspond pas à une remise en culture de parcelles anciennes, il s'agit parfois de sols pauvres ou à risques. Aussi, compte tenu de l'évolution de l'occupation spatiale des villes et de leur histoire, nous proposons de considérer avec attention la manière dont les dimensions environnementales et sanitaires entrent en compte dans la valorisation des productions « locales ». Prenons un dernier exemple : depuis le $\mathrm{xxI}^{\mathrm{e}}$ siècle, la ville de Paris s'est assainie en rejetant ses gadoues et ses boues sur sa périphérie. Ces déchets étaient soit enfouis dans ce qu'on appelait les " puits d'absorption ", soit épandus sur les terres maraîchères (Barles, 1999, op. cit.). Sous la pression de l'urbanisation, les maraîchers ont été repoussés de plus en plus loin du centre urbain (Phlipponeau, 1956). Deux solutions s'offraient à eux : soit ils partaient avec leurs terres, soit ils les revendaient à la Ville de Paris qui en avait besoin pour réaliser ses espaces verts. Des analyses du sol montrent que les plus anciens jardins parisiens ont été contaminés avec ces terres rapportées (Canolle, 2016). On a donc réimporté les pollutions qu'on avait autrefois rejetées à l'extérieur, sans oublier qu'entre-temps les maraîchers ont beaucoup utilisé de pesticides sur ce type de cultures. Le local n'est donc pas une ressource écologique toujours pertinente si on prend en compte l'historique du sol (péri)-urbain et l'héritage qui le constitue (Gitton et al., 2018).

L'analyse des discours sur les modes de production des connaissances expertes et de leurs usages invite à adopter une posture de réflexivité vis-à-vis du bien-fondé des échelles d'action. Il s'agit de cette manière de considérer les échelles, le local comme le global, comme le résultat de rapports sociaux, mais aussi comme liées aux formes de circulation des connaissances et de rapports de force. Le local n'est ni intrinsèquement bon, comme le global n'est ni par essence mauvais ; ils ne sont pas non plus figés, mais évoluent de manière relationnelle l'un par rapport à l'autre (Born et Purcell, 2006). Ce faisant, se focaliser sur la relocalisation peut limiter la portée d'enjeux globaux comme ceux qui traversent les systèmes alimentaires (Mormont, 2011). En revanche, rendre 
visibles les modes de production et de circulation des savoirs experts qui légitiment une échelle plutôt qu'une autre témoigne du rôle de la promotion du développement durable sur les valeurs positives attribuées au local (Lagagnier, 2002). On en vient alors à interroger la question des territoires pertinents pour nourrir la ville de demain. Sans remettre en question un besoin de réponse à la demande croissante de traçabilité de la part des consommateurs, ni le principe de réduction des distances entre lieux de production et de consommation, le débat porte sur la montée en puissance de la prise en compte de la qualité physico-chimique des sols lorsqu'on se rapproche des villes ${ }^{48}$.

\section{Conclusion}

La promotion de projets d'AU peut conduire à une perte de repères sur la connaissance $\mathrm{du}$ sol en ville au moment même où celui-ci commence à émerger sur la scène publique. Dans les différentes configurations étudiées, le jeu dialectique autour de la production de savoirs/ignorance sur la nature des sols (péri)urbains et les risques qui lui sont associés nous conduit aux résultats suivants.

On croyait s'être débarrassés des déchets en considérant le sol comme un filtre gratuit et commode (Barles, 1999, op. cit.). Pourtant le sol n'oublie rien et rappelle à notre bon souvenir les mésusages passés qui l'ont autrefois souillé et le corrompent encore. L'écologisation des pratiques concerne le geste humain, mais peut amener à minimiser la qualité du sol alors que celle-ci est supposée être le socle fondateur de toute pratique écologique. Le sol demeure un actant (Latour, 1994) « rancunier ». L'analyse des risques pose également une dimension temporelle $: \mathrm{si}$ « dans la société du risque, le passé perd de sa fonction déterminante pour le présent. C'est l'avenir qui vient s'y substituer » (Beck, 2001 : 61), le sol réactive, quant à lui, avec force le passé. Apparaît en effet l'importance de la question des héritages urbain et industriel, mais aussi agricole et jardinier qui l'affectent durablement. Plus encore, au nom du développement des cultures dans le sol urbain et de la valorisation du local, on favorise paradoxalement le hors-sol, que ce soit en réalisant des jardins coupés du sol en place ou en tentant de développer des tours verticales en hydroponie déconnectées du sol, mais aussi de l'air et du soleil, conduisant ainsi à l'artificialisation accrue du vivant.

41 En effet, au terme de ce travail, et en dépit des campagnes de communication sur l'agriculture dite urbaine apparait un point inattendu. Parler de "hors-sol bio» apparait comme un oxymore dans la mesure où il peut contribuer à produire de l'opacité. Qui plus est, ces cultures sans sol considèrent le sol reconstitué comme un support de culture orienté seulement vers la nourriture des végétaux cultivés ; si cette forme de culture peut lutter contre la rareté des sols cultivables (Donadieu et al., 2016), elle entre parfois en concurrence avec les terres agricoles périurbaines - comme l'illustre la controverse autour du triangle de Gonesse ou celle du plateau de Saclay ${ }^{49}$. Ce type de culture se caractérise aussi par une vision de l'ingénieur (bien loin de la figure de l'agriculteur ou du jardinier) contrôlant l'essentiel des facteurs de production. Enfin, le consommateur n'est pas informé qu'il achète des tomates hydroponiques, cultivées sur un substrat inerte et alimentées au moyen de «solutions nutritives », c'est-à-dire, pour notre propos, sans sol.

42 Le sol est, pour le moment, pris dans cette difficulté à revenir en arrière pour traiter les problèmes que l'on a mis précédemment sous l'éteignoir (jardins près d'autoroutes, sur des délaissés urbains potentiellement problématiques ou du moins qui mériteraient 
une vigilance) et une « urgence », selon les experts du développement, à développer de l'alimentaire en zones urbaines et périurbaines, bien qu'elles soient en général plus polluées que les zones agricoles. Ce qui change, c'est qu'aujourd'hui on sait, de plus en plus, du moins dans la sphère experte et gestionnaire que la situation en milieu urbain peut être problématique, et que cette contamination peut se transférer aux fruits et légumes cultivés (Potex, op. cit.; Dron, 2009). Ce débat d'experts ne touche pas encore un large public, même si des restitutions sont organisées dans les jardins collectifs concernés pour prévenir les jardiniers lorsque des contaminations sont découvertes dans leur parcelle (Canavese et al., 2016).

Un rapport différent à l'incertitude régit alors les exemples cités précédemment que l'on peut résumer par les deux tendances suivantes: une réduction de l'incertitude pour agir dans l'urgence au risque de produire du faux ou de taire certains problèmes, car le moment de la production de connaissances se confond avec celui de l'action versus une incertitude ayant une vertu heuristique et s'ouvrant vers les publics concernés. C'est bien la culture de l'urgence qui est questionnée dans ces propos d'U. Beck (2001: 62) : « Tout d'abord, les risques constituent des biens qu'il s'agit d'éviter, dont on soutient la non-existence jusqu'à ce que l'on soit contredit - en vertu du principe selon lequel en cas de doute, il faut préférer le progrès, ce qui équivaut à dire que, en cas de doute, mieux vaut détourner les yeux $»^{50}$.

La méconnaissance du sol urbain inviterait plutôt à ralentir (Stengers, 2017, op. cit.) et à prendre du recul sur les premières expériences menées pour en tirer des enseignements au risque de provoquer des « effets boomerangs " (Beck, 2001) que l'on ne pourrait plus feindre d'ignorer. Car adopter l'idée du sol urbain nourricier conduit inévitablement à une fuite en avant dans une gestion techniciste des risques qui demeure complexe et incertaine. Celle-ci peut participer à la production de l'ignorance dans la mesure où l'on risque de créer de nouveaux problèmes (à commencer par la santé de l'agriculteur ${ }^{51}$ lui-même travaillant sur de tels sites) en allant cultiver sur des sols de plus en plus contaminés sans avoir prévenu le consommateur de la construction d'une filière alimentaire dans de telles conditions environnementales et sanitaires ${ }^{52}$.

L'écologisation des politiques publiques au niveau local participe à la mise en valeur de modèles agricoles urbains dont il s'agit d'analyser les critères de la durabilité. Pour autant, on constate que leur usage des sols implique une étude attentive de l'ensemble des ressources nécessaires à la production. Considérer d'emblée la proximité comme une forme d'écologisation des pratiques agricoles et des systèmes alimentaires est une approche certes intéressante, mais non suffisante. L'écologisation des systèmes agrialimentaires implique une approche holistique du système de production et de consommation qui ne peut se réduire à de bonnes pratiques ni à une simple question d'échelle spatiale et de localisation par rapport à la ville.

Au terme de ce travail, on constate une lutte " épistémique » entre différents experts, d'une part des scientifiques cherchant à caractériser le sol pour définir ex post ses usages - jouant même un rôle de sensibilisation sur la question de la qualité des sols et quelques scientifiques très visibles car liés à des entreprises qui ont un intérêt économique direct au développement des projets ou un intérêt indirect «lorsque l'espérance que porte la science se confond ou se dilue dans un jeu de séduction avec ses effets d'annonce pour capter des crédits ou des postes " (Coutellec, op. cit. : 54).

L'ignorance est certes d'abord le produit d'un travail de communication vers le public profane, mais aussi d'un travail d'influence sur la connaissance elle-même pour décider 
des potentialités du milieu urbain. Ce qui passe, dans certains cas, par une sousestimation des risques qui lui sont associés. Cette dialectique est aussi repérable vis-àvis de conseils apportés auprès des organismes publics : à titre d'exemple lors d'une réunion avec un acteur administratif au printemps 2019, un expert appartenant à cette seconde catégorie préconise de ne pas prévenir les habitants de la qualité des sols lorsqu'il cultive des légumes au pied de leur immeuble afin de ne pas les affoler inutilement, faisant la supposition que la consommation dans ces jardins est souvent modeste. Cela pose question: en effet, il a été reconnu que, même sur de petites surfaces, des jardiniers peuvent produire et consommer une quantité non négligeable de légumes (Pourias, op. cit.). De surcroît, cet arbitrage (biaisé par la défense d'intérêts économiques et de quête de prestige) ne permet pas aux habitants de se positionner sur la question de la pollution des sols en toute connaissance de cause.

Dans ce contexte, un fossé peut se creuser entre ceux qui définissent les risques et ceux qui les subissent, entre ceux qui ont accès au savoir et ceux qui en dépendent (Beck, op. cit. : 84), créant ainsi des inégalités sociales qui mériteraient d'être approfondies ${ }^{53}$. Ne serait-ce pas le moment de se donner pour tâche d'expliciter les attachements, intérêts et valeurs (Coutellec, $2015: 54$ ) de ceux qui élaborent les outils et les principes par rapport auxquels les risques sont évalués, en laissant une place à la subjectivité qui les anime pour aller vers une appréciation commune des enjeux politiques, éthiques et culturels liés à la connaissance des sols (péri)urbains? La question ne concerne pas la persistance de zones d'ignorance - comme l'ont montré les historiens des sciences Oreskes et Conway: "Il y a toujours des incertitudes dans une science vivante, parce que la science est un processus de découverte» (2012:61) - mais elle renvoie davantage à la nécessité de comprendre pourquoi l'« on ignore ce que l'on sait?» (Dedieu et Jouzel, 2015). Par rapport au développement de ces technosciences, quelle exigence pourraiton alors adopter en situation d'incertitudes afin d'intégrer à leurs démarches «les éventuels effets induits prétendument invisibles» (Beck: 346). Car, comme le souligne encore (Beck : 67), « les risques finissent tôt ou tard, par atteindre ceux qui les produisent ou en profitent $»$.

\section{BIBLIOGRAPHIE}

Ademe, Villatte M., 2017, L'agriculture urbaine, quels enjeux de durabilité ?, 24 p., https://

www.ademe.fr/sites/default/files/assets/documents/agriculture_urbaine.pdf.

Association Robin des bois, 2016, Atlas de la France toxique, Paris, Flammarion, 164 p.

Baysse-Lainé A., Perrin C., 2017, « Les espaces agricoles en circuits courts de proximité : une lecture critique de la relocalisation de l'approvisionnement alimentaire de Millau. », Natures Sciences Sociétés, vol. 25, n 1, p. 21-35., https://doi.org/10.1051/nss/2017017.

Barles S., Breysse D., Guillerme A. et Leyval C., 1999, Le sol urbain, Paris, Anthropos.

Barles S., 1999, La ville délétère. Médecins et ingénieurs dans l'espace urbain. XVIII ${ }^{e}-X I X^{e}$ siècle, Paris, PUF, $336 \mathrm{p}$.

Développement durable et territoires, Vol. 11, $\mathrm{n}^{\circ} 1$ | Avril 2020 
Beck C., 2019, La qualité des sols aussi a une histoire, Journée d'échanges « Qualité des sols urbains et périurbains et compatibilité des usages, » dans le cadre du GT « Risques liés aux jardins collectifs et privatifs urbains ", Pollusols, LabEx Basc, Fire, Paris, 13 mai 2019.

Beck U., 2001, La société du risque : sur la voie d'une autre modernité, Paris, Aubier, 521 p.

Billet P., 2018, « Le statut juridique des sols face à l'artificialisation : état des lieux et perspectives ", Responsabilité et environnement, Paris, Annales des mines, n 91, p. 24-28, 97 p.

Born B., Purcell M., 2006, « Avoiding the Local Trap : Scale and Food Systems in Planning Research », Journal of Planning Education and Research.

Branchu P., Badin A.-L., Bechet B., Eisenlohr L., Le Priol T., Marseille F., Trielli E., 2012, « Pollution d'origine routière et environnement de proximité ", Vertigo - la revue électronique en sciences de l'environnement, hors-série ; DOI : http://doi.org/10.4000/vertigo.12775.

Brondeau F. (dir.), 2017, « Cultiver la ville : de la diversité des pratiques et des formes d'appropriation citoyennes », numéro spécial, Paris, L'Harmattan, nº 101, p. 119-138.

Brun M., 2015, Biodiversité végétale et délaissés dans l'aménagement urbain - Contribution potentielle des délaissés urbains aux continuités écologiques, thèse de doctorat, université de Tours, $480 \mathrm{p}$.

Bouquet D., Canavese M., Rémy É., Lebeau T., 2017, « Les jardins familiaux des Églantiers : la recherche à l'épreuve du "plein air" ». Les Journées du risque 2017, Le risque environnemental, Regards interdisciplinaires et nouvelles formes de régulation, 15 et 16 novembre 2017 - Terra Botanica Angers.

Callon M., Lascoumes P., Barthe Y., 2011, Agir dans un monde incertain. Essai sur la démocratie technique, Paris, Seuil, coll, « La couleur des idées », 358 p.

Canavese M., 2015, « "Vivre avec” les contaminations urbaines dans les jardins collectifs lillois : le point de vue des jardiniers ", rapport ISA Lille, projet de recherche ANR Jassur.

Canavese M., Bouquet D., Berthier N., Grenet M., Lebeau Th., Béchet B., Le Guern C., Douay F., Pinte E., Branchu Ph., Cambier Ph., Rémy É., 2016, « La qualité des sols urbains entre en politique ; du désordre aux nouveaux agencements sociotechniques dans les jardins collectifs urbains ", colloque Ordres et désordres au jardin, université de Strasbourg.

Canolle E., 2016, « Aménagement des espaces verts parisiens : évolution des mesures de caractérisation des sols et de leur qualité ", master 2 sciences de l'Univers, de l'environnement et de l'écologie, parcours géochimie environnementale, UPMC, $37 \mathrm{p}$.

Chabbal J., 2005, « Le risque invisible. La non-émergence d'un problème public », Politix, $\mathrm{n}^{\circ} 70$, p. 169-195, https://doi.org/10.3917/pox.070.0169.

Chalmandrier M., Canavese M., Petit-Berghem Y., Rémy É., 2017, « “L'agriculture urbaine”, entre concept scientifique et modèle d'action. Une notion mise à l'épreuve par le jardinage et le sol urbains », Géographie et cultures, n 101, DOI : https://doi.org/10.4000/gc.5052.

Chapelle G., 2013, «L'incidence des pollutions urbaines sur les productions alimentaires en ville », rapport final de la recherche réalisée pour le compte de l'Institut bruxellois de gestion de l'environnement, Bruxelles, Greenloop, 35 p.

Chateauraynaud F., Torny C., 1999, Les sombres précurseurs, Paris, EHESS, 476 p.

Conseil départemental du Val-de-Marne, 2018, «Plan d'action pour une agriculture en transition ", DEFIS_DEVP, 29 p.

Cauuet B., Domergue C., Urteaga M., 2005, « Mines et métallurgie en Aquitaine et en Hispanie septentrionale sous les Julio-Claudiens » in L'Aquitaine et l'Hispanie septentrionale à l'époque julio- 
claudienne. Organisation et exploitation des espaces provinciaux, colloque Aquitania, Saintes, 11-13 septembre 2003 ; Bordeaux, 2005, p. 423-460 (Aquitania, supplément, 13).

Coutellec L., 2015, La science au pluriel. Essai d'épistémologie pour des sciences impliquées, Versailles, Quae, $88 \mathrm{p}$.

Daniel A.-C., 2014, « Les terres d'apport d'île-de-France », rapport ANR Jassur.

Darly S., Demailly K., 2017, « Urban agriculture on the move in Paris : the routes of temporary gardening in the neoliberal city », ACME, p. 332-361.

Dedieu F., Jouzel J.-N., 2015, « Comment ignorer ce que l'on sait ? La domestication des savoirs inconfortables sur les intoxications des agriculteurs par les pesticides ", Revue française de sociologie, Paris, Presses de Sciences Po, vol. 56, n 1, p. 105-133, https://doi.org/10.3917/rfs. 561.0105

Demailly K., 2015, Jardiner les vacants. Fabrique, gouvernance et dynamiques sociales des vacants urbains jardinés du nord-est de l'île-de-France, thèse de doctorat en géographie, Université Paris I, $436 \mathrm{p}$.

Demailly K., 2014, «Les jardins partagés franciliens, scènes de participation citoyenne ? », EchoGéo, $\mathrm{n}^{\circ}$ 27, https://doi.org/10.4000/echogeo.13702.

Donadieu P., Rémy É., Girard M.-C., 2016, « Les sols peuvent-ils devenir des biens communs ? », Natures Sciences Sociétés, vol. 24, n 3, p. 261-269, https://doi.org/10.1051/nss/2016025.

Douay F., Roussel H., Pruvot C., Waterlot C., 2008, « Impact of a smelter closedown on metal contents of wheat cultivated in the neighbourhood ", Environmental, Science and Pollution Research, $\mathrm{n}^{\circ} 15$, p. 162-169.

Dron C., 2009, « Résultats et enseignements des actions mises en œuvre en 2008 sur le site des "Murs à pêches" de Montreuil-sous-Bois visant à caractériser la contamination des sols par les éléments traces métalliques (ETM) et à appréhender l'impact sur la conformité réglementaire des productions maraîchères et fruitières ", rapport Driaff.

Duchemin É. (dir.), 2013, « Agriculture urbaine : aménager et nourrir la ville », Éditions en environnement Vertigo, 394 pages.

Dubost F., 1997, Les jardins ordinaires, Paris, L'Harmattan.

Fillion E., Torny D., 2016, « Un précédent manqué : le Distilbène et les perturbateurs endocriniens. Contribution à une sociologie de l'ignorance ", Sciences sociales et santé, vol. $34, \mathrm{n}^{\circ} 3$, p. 47-75, https://doi.org/10.3917/sss.343.0047.

Frioux S., Canavese M., 2019, « Les sols pollués, un risque discret ? Le cas de l'agglomération lyonnaise ", Écologie \& Politique, n 58, Paris, Presses de Sciences Po, p. 53-68.

Gautier D., Benjaminsen Tor A., 2012, Environnement, discours et pouvoir, Versailles, Quæ, 256 p.

Ginelli L., 2012, « Chasse-gestion, chasse écologique, chasse durable... Enjeux d'une écologisation ", Économie rurale, $\mathrm{n}^{\circ}$ 327-328, http://economierurale.revues.org/3342.

Ginelli L., 2017, Jeux de nature, natures en jeu. Des loisirs aux prises avec l'écologisation des sociétés, Bruxelles, Peter Lang.

Gitton C., Verger Y., Brondeau F., Charvet R., Nold F., Branchu P., Douay F., Lamy I., Mougin C., Petit C., Rémy E., 2018, « L'économie circulaire : cercle vertueux ou cercle vicieux ? Le cas de l'utilisation de terres maraîchères pour aménager des espaces verts urbains ", Géocarrefour, vol. 92, nº 2, https://doi.org/10.4000/geocarrefour.11950. 
Girel M., 2013, « Agnotologie : mode d'emploi », Critique, 12, n 799, p. 964-977.

Girel M., 2017, Sciences et territoire de l'ignorance, Versailles, Quæ, 156 p.

Gis Sol, 2011, L'état des sols de France. Groupement d'intérêt scientifique sur les sols, 188 p.

Grenet M., Rémy E., Canavese M., Berthier N, 2016, « Des jardiniers à l'épreuve du sol urbain, L'exemple de jardins collectifs en France », Projets de paysage, http://www.projetsdepaysage.fr/fr/ des_jardiniers_1_preuve_du_sol_urbain.

Hughes T. P., 1986, « The Seamless Web : Technology, Science, Etcetera, Etcetera », Social Studies of Science, vol. 16, $\mathrm{n}^{\circ}$ 2, p. 281-292.

IAU IDF, 2015, « Des circuits courts aux filières de proximité. ", Les filières courtes de proximité au sein du système alimentaire francilien, fascicule $1,39 \mathrm{p}$.

Jacquier M., 2018, «Jardins collectifs urbains et contamination par les pesticides », rapport AgroparisTech.

Jouzel J.-N., Dedieu F., 2013, « Rendre visible et laisser dans l'ombre. Savoir et ignorance dans les politiques de santé au travail ", Revue française de science politique, vol. 63, n 1,p. 29-49, https:// doi.org/10.3917/rfsp.631.0029.

Kahle P., 2000, « Heavy metals in garden soils from the urban area of Rostock », Journal of plant Nutrition and Soil Science., vol. 163, n², p. 191-196.

Laganier R., Villalba B., Zuindeau B., 2002, « Le développement durable face au territoire : éléments pour une recherche pluridisciplinaire ", Développement durable \& territoires, dossier 1 , https://doi.org/10.4000/developpementdurable.774.

Lascoumes P., 1994, L'éco-pouvoir. Environnements et politiques, Paris, La Découverte, 318 p.

Latour B., 1989, La science en action, Paris, La Découverte.

Latour B., 1994, « Une sociologie sans objet ? Note théorique sur l'interobjectivité », Sociologie du travail, $36^{\mathrm{e}}$ année, $\mathrm{n}^{\circ} 4$, octobre-décembre « Travail et cognition », p. 587-607.

Letté M., Le Roux T. (dir.), 2013, Débordements industriels. Environnement, territoire et conflit XVIII ${ }^{e}-\mathrm{XXI}{ }^{e}$ siècle, Rennes, PUR, $402 \mathrm{p}$.

Mormont M., 2009, « Globalisations et écologisations des campagnes », Études rurales, $\mathrm{n}^{\circ} 183$, https://doi.org/10.4000/etudesrurales.8980.

Morel Journel C., Gay G. et Ferrieux C., 2018, « La résilience territoriale comme principe et comme volonté. Réflexions à partir de la question de la pollution des sols dans des territoires (dés)industrialisés ", Vertigo - la revue électronique en sciences de l'environnement, hors-série, DOI : http://doi.org/10.4000/vertigo.19172.

Ogé G., 2013, « Sols pollués et droits », in Euzen A., Eymard L., Gaill F., Le développement durable à découvert, Paris, CNRS Éditions, p. 246-247.

Gaill F. (dir.) Le développement durable à découvert, Paris, CNRS Éditions, 363 p.

Oreskes N., Conway E., 2012, Les marchands de doute, trad. de l'américain par Jacques Treiner, Paris, Éd. Le Pommier, coll. » Essais et documents », 524 p.

Paddeu F., 2015, De la crise urbaine à la réappropriation du territoire. Mobilisations civiques pour la justice environnementale et alimentaire dans les quartiers défavorisés de Detroit et du Bronxà New York, thèse de doctorat en géographie, Université Paris 4. 
Paddeu F., 2017, « Manger local. Leurres et promesses », Vacarme, vol. 81, n 4, p. 40-45, https:// doi.org/10.3917/vaca.081.0040.

Phlipponeau M., 1956, La vie rurale dans la banlieue parisienne, Paris, Armand Colin, 593 p.

Potex :POTagers Expérimentaux, 2016, « Synthèse de l'étude de la qualité chimique des végétaux de jardins partagés parisiens en fonction de différents aménagements visant à confirmer la pertinence des pratiques de gestion de la ville de Paris », Agence d'écologie urbaine.

Pourias J., 2014, Production alimentaire et pratiques culturales en agriculture urbaine : analyse agronomique de la fonction alimentaire des jardins associatifs urbains à Paris et Montréal, thèse, Montréal (Québec, Canada), université du Québec, doctorat en sciences de l'environnement.

Proctor R. N., 1996, Cancer Wars : How Politics Shapes What We Know and Don't Know About Cancer, Basic Books, 368 p.

Purseigle F., Poupart A., Compère P., 2012, « La ferme verticale : image paroxystique de mondes agricoles en mutation ", Agrobiosciences.

Rémy É., Branchu P., Canavese M., Berthier N., 2017, les risques sanitaires liés aux jardins collectifs : l'expertise sur le sol urbain en débat, Lien social et politiques, n 78, p. 49-69, https:// doi.org/10.7202/1039338ar.

Ronsin G., 2018, Composer les relations entre « science » et " gestion de la nature ». Ethnographie des frontières, casquettes et controverses dans les conseils scientifiques, thèse de sociologie, Grenoble, Université Grenoble Alpes.

Schwartz C., 1993, « Facteurs de qualité des sols de jardins de l'est-mosellan », mémoire DEA Sciences agronomiques, INPL, Ensaia, $44 \mathrm{p}$.

Slocum R., Blumberg R., Valentine Cadieux K., 2016, « Solidarité, espace et "race" : vers des géographies de la justice alimentaire " justice spatiale|spatial justice, $\mathrm{n}^{\circ} 9$.

Stengers I., 2017, «Que serait une science responsable », Sciences critiques, https://sciencescritiques.fr/que-serait-une-science-responsable.

Tixier C., Sancelme M., Aït-Aïssa S., Widehem P., Bonnemoy F., Cuer A., Truffaut N., Veschambre H., 2002, Biotransformation of phenylurea herbicides by a soil bacterial strain, Arthrobacter sp. N2 : structure, ecotoxicity andfate of diuron metabolite with soil fungi, Chemosphere, $n^{\circ} 46$, p. 519-526.

Tornaghi C., 2014, « Critical geography of urban agriculture », Progress in Human Geography, vol. 38, n 4, p. 551-567, https://journals.sagepub.com/doi/full/10.1177/0309132513512542.

Zanetti T., Morel-Journel C., Ferrieux C., Gay G. (dir.), 2018, « Visibilité et invisibilité de la pollution des sols dans les territoires (post)industriels », Géocarrefour vol. 92, n² 2, p. 551-567, https://doi.org/10.4000/geocarrefour.11289.

\section{NOTES}

1. Nous emprunterons des exemples relevant des jardins collectifs (jardins familiaux, jardins partagés) ou de différentes formes d'« agriculture urbaine » (agriculture hors sol, " micro-fermes urbaines ») - pour une discussion sur ces divers types de cultures en ville qui renvoient à des mondes sociaux et techniques fort différents, se reporter à Chalmandrier (et al.) 2017. 
2. Dion Cyril, Laurent Mélanie, Demain, film produit par Move Movie, sorti le 2 décembre 2015.

3. Les délaissés urbains peuvent se définir de la manière suivante, ce sont «des espaces dépourvus d'usage officiel, des lieux transitoires présentant, du fait d'une gestion irrégulière, voire inexistante, une végétation spontanée (Brun, 2015 ; Demailly, 2015)».

4. On peut citer l'action juridique engagée par France Nature Environnement contre la mise en culture du toit d'un réservoir d'eau de Paris. Cette association considère cet espace comme un "réservoir de biodiversité " à préserver dans un espace dense et s'oppose au développement de serres en plastiques sur un tel site.

5. Il s'agit principalement de scientifiques en pédologie, agronomie, sciences du sol, géochimie, chimie.

6. Fondateur de l'agnotologie, discipline qui étudie la production culturelle de l'ignorance.

7. Il s'agit donc dans cet article d'un exercice un peu différent de celui de nos publications précédentes, lesquelles mettaient davantage l'accent sur une analyse ethnographique de ces terrains (Grenet et al., Projets de paysage, 2016).

8. Pour plus de détails sur ces enquêtes de terrain : Lyon (de 2013 à 2017), Paris (de 2013 à aujourd'hui : 13 jardins et 43 jardiniers), Lille (en 2014: 7 jardins et 25 jardiniers) et Nantes (en 2014: 1 jardin familial et 37 jardiniers) à l'appui de divers projets de recherche : projet ANR Jassur, Revisol financé par le Labex IMU, projet Comete en lien avec l'Agence d'écologie urbaine Paris.

9. Les circuits courts ont été définis par le plan Barnier (2009) : «Un circuit court est un mode de commercialisation des produits agricoles qui s'exerce soit par la vente directe du producteur au consommateur, soit par la vente indirecte à condition qu'il n'y ait qu'un seul intermédiaire. » La loi d'avenir pour l'agriculture du 13 octobre 2014 considère en même temps le développement des "circuits courts et de la proximité géographique entre producteurs agricoles, transformateurs et consommateurs». Émerge une approche qui valorise à la fois la proximité sociale et géographique : la notion de circuits courts de proximité. Elle est notamment mise en avant par des acteurs publics comme l'institut d'aménagement et d'urbanisme de l'île-de-France (IAU IDF) : "Les filières courtes de proximité visent à créer des modes où l'humain, la viabilité économique et le local sont privilégiés. [...] Pour les filières courtes de proximité, la proximité géographique compte tout autant que la proximité relationnelle. » (IAU IDF, 2015, : 5).

10. Précisons qu'il existe différentes cultures épistémiques sur les sols portées par l'agronomie (elle-même très diverse et diversement positionnée sur le sujet), la pédologie, les sciences du sol, la géochimie, l'écotoxicologie qui n'impliquent pas le même rapport aux risques.

11. À savoir que les risques finissent par atteindre ceux qui les produisent.

12. Conscientes de l'absence d'une définition stable du terme "technosciences", nous l'utilisons pour qualifier la science contemporaine, dépendante des enjeux économiques et sociaux (Rollet, 2001 ; Bensaude-Vincent, 2009), et donc liée au monde des techniques. La nature de ce lien, parfois qualifié de «tissu sans couture » (traduction de la notion de "seamless web» (Hughes, 1986)) reste à interroger et à préciser dans le cas du local alimentaire, ce que nous tenterons de faire dans cet article. 
13. Précisons toutefois que pour certains chercheurs, le sol urbain est un objet d'étude pour la production de connaissances sur le mode de la science confinée (au sens que lui donne Callon op.cit.). Ce sont certains projets d'AU qui obligent, certains d'entre eux en tant qu'experts, à se positionner sur la qualité de ces sols en raison des usages sensibles qui y sont projetés.

14. Source communication personnelle à propos de la réunion AllEnvi du 29 novembre 2018.

15. Nous pouvons citer par exemple le cas de l'école de Vincennes: http:// www.leparisien.fr/societe/fermeture-de-l-ecole-franklin-roosevelt-de-

vincennes-14-06-2001-2002234890.php (consulté le 28 mars 2019); ou encore le cas médiatisé des murs à pêches de Montreuil : http://www.leparisien.fr/seine-saintdenis-93/montreuil-la-prefecture-impose-une-nouvelle-etude-d-impact-aux-murs-apeches-16-10-2018-7920387.php (consulté le 28 mars 2019).

16. C'est la raison pour laquelle les noms des sites ont été anonymisés. Rappelons toutefois que les teneurs totales en métaux des jardins collectifs sont deux fois supérieures à celles des sols agricoles (Schwartz, 1993 ; Kahle, 2000).

17. L'exposition "Capital agricole, Chantiers pour une ville cultivée " (on remarquera le Capital sans e qui prouve bien l'orientation technicoscientifique et économique envisagée) s'est déroulée au Pavillon de l'Arsenal (Paris $4^{\mathrm{e}}$ ) du $1^{\mathrm{er}}$ octobre 2018 au 17 février 2019.

18. Sur ce point nous rejoignons le propos d'Emmanuelle Fillion et Didier Torny (2016, op. cit.) selon lesquels la connaissance des profanes est "systématiquement considérée comme une source de panique et non d'action raisonnée ».

19. C'est la raison pour laquelle nous utilisons le terme de technoscience en référence à Stengers (2017) pour décrire ce type de recherche qui est mise directement au service de projets d'AU portés par l'agro-industrie ou des groupes immobiliers. Les usages attribués à ces sols sont décidés a priori alors que les connaissances scientifiques sont peu stabilisées. L'absence de prise de recul sur les premières expériences associée à la culture de l'urgence peut poser problème; il faut en effet disposer de temps pour acquérir et valider les données, suivre les effets de conseils apportés hors de l'enceinte simplifiée du laboratoire et les réorienter si besoin lors d'une expérimentation en vraie grandeur qui apportera inévitablement son lot d'aléas (météorologiques, écart entre les données de la littérature et les données de terrain, processus inattendus, etc.).

20. Dans les évolutions récentes, on peut noter aussi à la présidence d'une association locale de défense de l'environnement en région Auvergne-Rhône-Alpes, une personne référente sites et sols pollués au sein du réseau France Nature Environnement, et siégeant au groupe de travail sites et sols pollués du Conseil supérieur de la prévention des risques technologiques (CSPRT).

21. Beaucoup de ces articles concernent le contexte parisien, comme c'est le cas des publications d'Enlarge your Paris: http://enlargeyourparis.blogs.liberation.fr/ 2018/10/21/le-grand-paris-est-un-parfait-laboratoire-agricole/ (consulté le 21 octobre 2018) ou d'articles parus dans Le Parisien : http://www.leparisien.fr/paris-75005/ parisculteurs-du-reve-a-la-recolte-27-09-2017-7290846.php (consulté le 3 avril 2019). On trouve néanmoins des publications concernant d'autres métropoles comme Marseille (https://www.20minutes.fr/planete/2257687-20180418-marseille-retouragriculture-urbaine-70-ans-apres-ville-autosuffisante (consulté le 2 avril 2019) ou 
encore Rennes (https://france3-regions.francetvinfo.fr/pays-de-la-loire/loireatlantique/nantes/agriculture-urbaine-villes-retrouvent-chemin-terre-1463123.html (consulté le 2 avril 2019).

22. On peut citer quelques exemples d'articles parus dans la presse économique consacrés aux perspectives ouvertes par les start-up de l'AU: Dans le magazine L'Expansion https://lexpansion.lexpress.fr/entreprises/agricool-les-aventuriers-de-lagriculture-urbaine_1940915.html (consulté le 2 avril 2019) ou encore dans les colonnes des Échos https://business.lesechos.fr/entrepreneurs/innovation-recherche/ 030769030023-agricool-topager-les-start-up-misent-sur-l-agricultureurbaine-315291.php (consulté le 26 mai 2018).

23. Plusieurs articles sur les «utopies d'écologie hors sol» sont parus dans des magazines spécialisés sur les problématiques socio-écologiques comme Basta! Mag (https://www.bastamag.net/L-agriculture-urbaine-hors-sol-solution-miracle-ouencouragement-a-la (consulté le 20 mars 2018), Usbek et Rica qui relaie les paroles d'un agriculteur urbain sceptique sur les bénéfices écologiques des techniques hors sol: https://usbeketrica.com/article/les-fermes-verticales-ou-l-utopie-d-une-ecologiehors-sol (consulté le 7 novembre 2018); Ou encore le magazine du réseau de distribution La Ruche qui dit Oui !: https://magazine.laruchequiditoui.fr/cultures-sanssol-et-sans-reproches/ (consulté le 3 août 2018).

24. Exemple des sociétés Sous les fraises (https://www.youtube.com/watch? $\mathrm{v}=$ sXllQeZ-8-o (consulté le 15 mai 2018), Agricool et les Sourciers: https:// www.youtube.com/watch?v=_-whcTeyk64 (consulté le 3 avril 2019).

25. Pour l'entreprise implantée dans les Yvelines, La boîte à champignons, les 689536 kilogrammes de marc de café récoltés pour la culture de pleurotes entrent dans le calcul de "l'impact positif » de ce mode de culture, en participant notamment à la " régénération de plus de $19477 \mathrm{~m}^{2}$ de sol » (https://www.laboiteachampignons.com/ (consulté le 3 avril 2019). Ces éléments sont communiqués à des politiques (comme ici les ministres de l'Agriculture et de la Santé : https://actu.fr/ile-de-france/saint-nomla-breteche_78571/saint-nom-breteche-deux-ministres-visitent-boite-

champignons_13363677.html) et sur le site de l'entreprise. Pourtant, ni la manière dont ces chiffres sont calculés ni les moyens par lesquels les sols ont été "régénérés » ne sont explicités dans ces discours de communication.

26. Notons que la qualité des composts réalisés par les amateurs, les collectivités, etc. est peu connue et fait l'objet d'un projet de recherche en cours, dénommé Potagers, et piloté par Aurélie Pelfrêne (ISA Lille).

27. «Les Parisculteurs» est un dispositif mis en place par la Mairie de Paris dans le cadre des objectifs établis par la municipalité dans la charte "Objectif 100 hectares ", signée en 2016. Elle vise la végétalisation de 100 hectares de bâti (façades, murs et toits), dont 33 hectares d'agriculture urbaine. Sous la forme d'appels à projets, les candidats à l'installation en agriculture sont invités à postuler sur des sites mis à disposition par des acteurs publics et privés. En 2019, la troisième session d'appels à projet est lancée. À des sites dans Paris intra-muros sont désormais associés des sites dans la petite couronne parisienne. À la différence des éditions précédentes, près des « deux tiers » des sites mis au concours sont « au sol» (site des Parisculteurs, consulté le 3 avril 2019). Pour plus d'informations sur le dispositif: http:// www.parisculteurs.paris/

28. Souligné par nous. 
29. À ce sujet, voir aussi les travaux du projet de recherche Ittecop.

30. Comme le souligne cette étude sur l'historique du site (Potex, 2016), l'usine fut exploitée de 1836 à 1937, puis détruite, probablement sans aucune mesure de protection conformément aux pratiques de l'époque - nous remercions Jeanne Pourias pour nos enquêtes réalisées en commun sur ce cas d'étude - voir aussi thèse de Jeanne Pourias (2014).

31. La variabilité temporelle et spatiale qui caractérise les mesures des polluants volatils rend impossible une évaluation fiable de leur étendue dans le sous-sol.

32. Une autre préoccupation des services techniques porte sur la provenance des terres rapportées, leur qualité et leur traçabilité - se reporter à Daniel (2014).

33. Se pose la question de la durabilité de la géomembrane, généralement garantie pour une quarantaine d'années. Que fait-on ensuite?

34. Souligné par nous.

35. Bien évidemment la connaissance du site (et de son aménagement) diffère d'un jardinier à l'autre en fonction de leur présence et de leur implication dans la vie du jardin, et du fait bien connu que l'information se transforme en passant de la bouche de l'un à l'oreille de l'autre. Il y a aussi un décalage dans le niveau d'information entre les premiers arrivants qui ont participé au projet d'origine et ont vu le jardin se construire, et ceux qui s'y insèrent progressivement.

36. Ce cas est loin d'être isolé, puisque la philosophie d'action des services techniques de la Ville de Paris est de ne pas cultiver dans le sol en place compte tenu de sa contamination, cf. Projet Potex, op. cit.

37. Nous pensons notamment aux enjeux fonciers et juridiques à parler de pollutions d'un site, le sol, contrairement à l'air et l'eau, étant toujours approprié. C'est la raison pour laquelle Frédéric Ogé avait formulé l'expression de sol " potentiellement pollué ", pour protéger juridiquement les personnes susceptibles de s'intéresser aux risques associés aux sols industriels ou anciennement industriels.

38. Pour certains agronomes, pédologues, géochimistes avec lesquels nous avons échangé ces cinq dernières années, le sol urbain a longtemps été considéré comme un non-sol et fait l'objet de débats sur ses usages actuels. D'autres considèrent qu'un technosol n'est pas un sol.

39. Source : M. Isambert, liste de diffusion AFES, 2017.

40. Source : https://montreal.lufa.com/fr et visite sur place le 24 août 2018.

41. L'usage du terme "ferme " prête à confusion. Il introduit l'idée de pratiques plus traditionnelles que les représentations accolées au terme d'" exploitation agricole ", alors même que dans les projets les plus technophiles ces producteurs ont recours à de nombreuses technologies (exemple : production sans soleil et sans sol).

42. Suivant le même principe, on peut citer aussi l'exemple du projet Farmbot: carré potager entièrement robotisé.

43. Source : https://montreal.lufa.com/fr/fermes, consulté le 31 août 2018.

44. Source: https://www.peasandlove.com/la-technique-de-culture (consulté le 3 avril 2019).

45. Source : communiqué de presse étude Peas\&Love publié sur la liste de diffusion de l'AFES, 30 août 2018. Citons un autre exemple : sur un petit marché breton en 2016, un 
petit producteur annonçait sur une pancarte «Ici tomates cultivées en pleine terre » affichant ainsi son opposition aux cultures hors sol.

46. "Production hydroponique» : cette méthode de culture consiste à placer les racines des végétaux dans une solution d'éléments nutritifs minéraux uniquement ou dans un milieu inerte, tel que perlite, graviers, laine minérale. Source : http://eurlex.europa.eu/legal-content/FR/TXT/HTML/?uri=CELEX: 02008R0889-20160507\&qid=1465559502531\&from=FR.

47. Source : http://www.fnab.org/images/stories/espace_presse/ CP_R\%C3\%A8glementation_Bio_lignes_rouges_09062017.pdf.

48. En ce sens, on note que le récent Plan d'action de l'agriculture en Val-de-Marne (2018) indique qu'il favorisera l'installation de nouveaux jardins partagés et familiaux à l'appui de ces trois conditions: "Vérifier la qualité des sols, diffuser les règles de santé publique de non-culture des sols pollués, accompagner les dynamiques associatives » (p. 14).

49. Débats dont la presse s'est fait l'écho comme dans cet article de Bastamag : https:// www.bastamag.net/L-agriculture-urbaine-hors-sol-solution-miracle-ouencouragement-a-la, consulté le 20 mars 2018.

50. Souligné par nous.

51. On pourrait ici faire un parallèle avec les travaux de Jouzel et Dedieu cités dans l'article au sujet de l'exposition des agriculteurs aux pesticides: dans les projets d'AU sur sol pollué, il est envisagé que l'agriculteur porte des gants et un masque. Toutefois, on peut émettre l'hypothèse qu'un tel équipement sera difficile à porter en zone périurbaine, puisque l'agriculteur se retrouvera confronté au regard des habitants; ce faisant, il risque de ne pas porter son équipement de protection et sera tenu pour responsable en cas de problème de santé pour n'avoir pas respecté les consignes suivant un effet pervers qui évite de poser la vraie question: est-ce une nécessité de cultiver de l'alimentaire sur des sols impropres? Dans le même ordre d'idées, Corinne Beck précise, dans une journée d'échanges tenue récemment (2019), que les archéologues urbains, confrontés à la pollution des sols lors de projets de rénovation urbaine, ne peuvent pas porter leur équipement de sécurité, car ils sont confrontés au regard des citadins s'indignant de voir des bâtiments se construire sur des sites pollués.

52. Travail en cours mené avec des économistes comme Sylvie Lupton et Yoann Verger dans le cadre d'une étude en cours sur les risques liés aux jardins collectifs urbains.

53. En guise d'illustration, précisons que le savoir sur les sols est médié par des instruments (sauf pollutions visibles), mais l'accès à ces analyses de sol n'est pas aisé et coûteux, ce qui peut être une source d'inégalités entre jardiniers ne disposant pas des ressources financières nécessaires; de plus, en raison du prix du foncier, les dépollutions en centre-ville sont mieux réalisées que dans les banlieues avoisinantes créant ainsi des inégalités socio-spatiales. 


\section{RÉSUMÉS}

L'engouement pour les circuits courts et de proximité pose la question de l'écologisation des systèmes agri-alimentaires. En ce sens, la qualité des végétaux cultivés sur les sols (péri)urbains interroge la société du risque et l'évaluation controversée qui en est faite. On assiste alors à une lutte de cadrages cognitifs et politiques par rapport à cette dialectique de production de savoirs/ ignorance sur les sols (péri)urbains. Plus encore, au nom du développement des cultures dans le sol urbain, on favorise paradoxalement le hors-sol, lequel soulève de nombreux questionnements sur le plan technique, anthropologique et politique. À partir d'enquêtes menées depuis plusieurs années, nous interrogeons dans cet article les mécanismes complexes qui produisent l'ignorance sur un nouvel objet émergent, à savoir le sol urbain nourricier valorisé au nom du primat du local.

The enthusiasm for short food supply chains questions agri-food systems' ecologisation. In that respect, the quality of plants growing on (peri) urban soils challenges the Risk Society and the controversial assessment of it. One can observe a struggle between cognitive and political frameworks in relation to this dialectic of knowledge production and ignorance on urban (peri) soils. Moreover, in the name of crops in urban soil development, cultivation off the ground is paradoxically chosen, which raises numerous technical, anthropological and political questions. Based on investigations carried out over several years, this article addresses the complex mechanisms that produce ignorance on an emerging object, that is, the nurturing urban soil valued in the name of local primacy.

\section{INDEX}

Mots-clés : local, cultures alimentaires, expertise, incertitudes, ignorance, sol (péri)urbain

Keywords : local, short food supply chains, expertise, uncertainties, ignorance, (peri) urban soil

\section{AUTEURS}

\section{ÉLISABETH RÉMY}

Élisabeth Rémy est sociologue à Inra-AgroParisTech. Elle travaille, depuis 2014, sur l'émergence controversée du sol urbain nourricier. De nombreux jardins collectifs (ou de projets d'agriculture urbaine) sont concernés par la contamination des sols (péri)urbains. Son travail vise notamment à analyser la dialectique de production de connaissances et d'ignorance autour de ces sols en lien avec les risques qui leur sont (ou non) attribués.

elisabeth.remy@agroparistech.fr

\section{NOLWENN GAUTHIER}

Nolwenn Gauthier est doctorante en socio-géographie. Elle rédige sa thèse à l'EHESS sur les politiques de territorialisation en Val-de-Marne au prisme de la justice alimentaire. no.gauthier@gmail.com

\section{MARINE CANAVESE}

Marine Canavese est doctorante en sociologie. Elle rédige sa thèse en codirection en histoire et sociologie au Centre Max Weber (Université Lyon 2). Elle écrit une sociohistoire des sols pollués 
et potentiellement pollués en milieu urbain en tant que problème public. Son travail de terrain se fait en archives nationales et locales (agglomération lyonnaise) ; mais aussi auprès des habitants qui sont confrontés à l'émergence du sol urbain à cause de sa pollution.

m.canavese@yahoo.fr 